ORIGINAL ARTICLE

\title{
Alterations of mononuclear inflammatory cells, CD4/CD8+ T cells, interleukin $1 \beta$, and tumour necrosis factor $\alpha$ in the bronchoalveolar lavage fluid, peripheral blood, and skin of patients with systemic sclerosis
}

\author{
M R Hussein, H I Hassan, E R M Hofny, M Elkholy, N A Fatehy, A E A Abd Elmoniem, \\ A M Ezz El-Din, O A Afifi, H G Rashed
}

J Clin Pathol 2005;58:178-184. doi: 10.1136/icp.2004.019224

See end of article for authors' affiliations

\section{Correspondence to:}

Dr M R Hussein,

Department of Pathology, Faculty of Medicine, Assiut University, Assiut, Egypt; mrh17@swissinfo.org

Accepted for publication 10 August 2004

\begin{abstract}
Background: Systemic sclerosis (SSc) is a multisystem disease with underlying immune mechanisms. Aims: To investigate the clinicopathological characteristics of the lesions; immunological alterations in the bronchoalveolar lavage fluid (BALF), peripheral blood, and skin; and correlations between the clinicopathological characteristics and immunological alterations in SSc.

Materials/Methods: Skin biopsies, BALF, and peripheral blood samples were obtained from 19 patients (18 women, one man) with SSc and six age and sex matched healthy controls (HCs). Mononuclear inflammatory cells (MICs), CD4/CD8 cells, tumour necrosis factor $\alpha$ (TNF $\alpha$ ), and interleukin 1 $\beta$ (IL1-1 $\beta$ ) concentrations were examined in all samples using histological methods, enzyme linked immunosorbent assay, and immunoperoxidase staining.

Results: The mean (SD) age of the patients with SSc was 34.8 (2.6) years. Proteinuria, positive rheumatoid factor, and $\mathrm{C}$ reactive protein were seen in $15.8 \%, 26.3 \%$, and $26.3 \%$ of patients, respectively. Compared with $\mathrm{HCs}$, there were significantly higher: total MICs (macrophages, lymphocytes), neutrophils, and eosinophils in BALF, blood, and skin (all $\mathrm{p}<0.05$ ); cytokine concentrations in BALF (TNF $\alpha, \mathrm{p}<0.001$; IL-1, $p<0.01$ ) and peripheral blood $(p<0.01$ and $p<0.05)$; and CD8/CD4+ T cells in peripheral blood $(p<0.05)$. Compared with $\mathrm{HCs}$, lesional skin had significantly higher histiocyte cell counts $(p<0.05)$, lower lymphocyte counts $(p<0.05)$, and higher $C D 4 / C D 8$ ratios $(p<0.001)$. There were significant correlations between cytokine concentrations and CD8+T cells and forced vital capacity $(p<0.001$ and $p<0.01$, respectively).

Conclusions: MICs, CD4/CD8+ cells, and cytokines are altered in SSc. These alterations correlated with the underlying disease process and therefore may have pathogenic, modulatory, and potential prognostic roles in SSc.
\end{abstract}

$\mathrm{S}$ ystemic sclerosis (SSc) is a multisystem autoimmune connective tissue disease that involves the skin, lung, kidney, and the heart. The pulmonary and cutaneous lesions include alveolitis and both cutaneous and pulmonary fibrosis. ${ }^{1}$ The pathogenesis of these lesions involves immunological alterations such as $\mathrm{T}$ cell activation, altered CD4/ CD8 ratio, and the recruitment of macrophages. These immune cells contribute to the release of several cytokines such as interleukin $1 \beta$ (IL- $1 \beta$ ), IL-2, IL-4, IL-6, and tumour necrosis factor $\alpha(\mathrm{TNF} \alpha) .{ }^{1} \mathrm{IL}-1 \beta$ is released by macrophages. It can stimulate $B$ cell growth, activate $\mathrm{T}$ cells, and induce acute phase protein production. It has inflammatory effects on fibroblasts and the endothelium. In addition, TNF $\alpha$ is released by macrophages, activated $\mathrm{T}$ cells, and natural killer cells. It activates both T cells and macrophages, in addition to inducing acute phase protein production. It has inflammatory effects similar to those of IL- $1 \beta .^{23}$

"We hypothesised that systemic sclerosis is associated with alterations in mononuclear inflammatory cells, T cell subsets, and cytokine production in the lung, blood, and skin"

Currently, our understanding of the immunological changes in patients with SSc is incomplete. In this investigation, we hypothesised that SSc is associated with alterations in mononuclear inflammatory cells (MICs), T cell subsets (CD4/CD8 ratio), and cytokine production in the lung, blood, and skin. We further hypothesised that these alterations can contribute to the genesis of the pulmonary and skin lesions and therefore should correlate with the clinicopathological characteristics of these lesions. We carried out our present investigation to fill the existing gap in the literature and to test our hypotheses. To this end, we examined 19 patients with SSc and six age and sex matched healthy individuals. Our specific aims included analysis of the patients with SSc for: (1) the clinicopathological characteristics of the lesions; (2) alterations in MICs, CD4/ CD8+ T cell subsets, and cytokine concentrations (IL-1 $\beta$ and $\mathrm{TNF} \alpha$ ) in the bronchoalveolar lavage fluid (BALF), peripheral blood, and skin; and (3) any correlations between the clinicopathological characteristics and the immunological alterations in these lesions.

\footnotetext{
Abbreviations: BAL, bronchoalveolar lavage; BALF, bronchoalveolar lavage fluid; CRP, C reactive protein; dSSc, diffuse systemic sclerosis; ECG, electrocardiogram; FVC, forced vital capacity HC, healthy control; $\mathrm{HRCT}$, high resolution computerised tomography; IL, interleukin; ISSc, limited systemic sclerosis; MIC, mononuclear inflammatory cell; PBS, phosphate buffer saline; SSc, systemic sclerosis; TGF $\beta$, transforming growth factor $\beta$; TNF $\alpha$, tumour necrosis factor $\alpha$
} 


\section{PATIENTS AND METHODS}

\section{Patients}

Nineteen patients with SSc ( 18 women and one man) were admitted to the departments of dermatology and rheumatology, Assiut University Hospitals School of Medicine, Assiut, Egypt (December 2002 to March 2004). According to the extent of the skin lesions, SSc was categorised into limited SSc (ISSc; 10 patients) and diffuse SSc (dSSc; nine patients). None of the patients was receiving immunosuppressive drugs at the time of our study. An additional six healthy individuals (healthy controls; HCs) were included. Full history and clinical examination were performed for all the participants. The diagnosis of SSc was rendered according to American College of Rheumatology criteria. ${ }^{4}$ Informed consent was obtained from all participants. The experimental design was approved by the ethics committee at the school of medicine, Assiut University, Assiut, Egypt.

\section{Assessment of the cardiopulmonary functions}

Chest $x$ ray, high resolution computed tomography, computerised spirometer (for spirometric pulmonary function tests including forced vital capacity (FVC), forced expiratory volume in the first second, peak expiratory flow, and forced expiratory flow at $25-75 \%$ of vital capacity), 12 lead electrocardiogram (ECG), 24 hour ambulatory ECG recording, and echocardiography were performed for all the participants. Left ventricular end diastolic dimensions, left ventricular end systolic dimensions, left ventricular systolic function (fraction shortening and ejection fraction) were measured in $\mathrm{M}$ mode. Left ventricular diastolic function (peak of early filling velocity ("E wave"), peak of late filling velocity ("A wave"), and E/A ratio) were measured by Doppler. Pulsed Doppler measurements of pulmonary valve systolic flow (pre-ejection time of right ventricle, time to peak velocity, and right ventricular ejection time) were also measured. Moreover, fibreoptic bronchoscopy with bronchoalveolar lavage (BAL) was performed to obtain BAL fluid (BALF).

\section{Assessment of the serological changes}

All the participants were evaluated for peripheral blood count, erythrocytic sedimentation rate, rheumatoid factor, C reactive protein (CRP), complete urine analysis, liver and kidney function tests, and antinuclear antibodies.
Isolation of MICs from the peripheral blood and BALF Fibreoptic bronchoscopy and BAL were performed using standardised techniques. Briefly, $6 \times 20 \mathrm{ml}$ aliquots of prewarmed normal saline were instilled into the lobe with the greatest parenchymal infiltrate. When infiltrates were symmetrically distributed, three subsegmental bronchi of the right middle lobe, right lower lobe, and lingula were sampled. The cells (macrophages, lymphocytes, neutrophils, and eosinophils) were separated from the BALF by centrifugation at $200 \times g$ at $4^{\circ} \mathrm{C}$ for seven minutes and washed twice with phosphate buffered saline (PBS). The cellular concentrations and recoveries were estimated using crystal violet staining. Cellular viability was measured using the trypan blue exclusion test. Differential cell counts were performed on 200 May-Grünwald-Giemsa stained cells, as described previously. ${ }^{5}$ For isolation of the lymphocytes, $5 \mathrm{ml}$ of peripheral venous blood and $10 \mathrm{ml}$ of BALF were collected into heparinised containers. The MICs of the peripheral blood and BALF were isolated on Ficoll Hypaque (high density gradient separation medium), as described previously. ${ }^{67}$ The MIC layers were washed three times with PBS, and then spun down. Several films were prepared for further cytological and immunohistochemical analysis (CD3, CD4, and CD8) using immunoperoxidase staining methods and monoclonal antibodies (T cells: anti-CD3, anti-CD4 (clone MT310), and antiCD8 (clone C8/144B); B cells: anti-CD20 (clone B-Lyl); all from DAKOCytomation, Carpinteria, California, USA), as described previously. ${ }^{8-10}$ The results (MIC counts) were expressed as a percentage.

\section{Measurement of TNF $\alpha$ and IL- $1 \beta$ concentrations in BALF and peripheral blood}

Aliquots ( $10 \mathrm{ml}$ ) of BALF were immediately centrifuged at $2000 \times g$ for 20 minutes at $4^{\circ} \mathrm{C}$ and the supernatants were stored at $-70^{\circ} \mathrm{C}$. TNF $\alpha$ and IL- $1 \beta$ concentrations $(\mathrm{pg} / \mathrm{ml})$ in the peripheral blood (serum) and BALF were measured using a solid phase, high sensitivity enzyme linked immunosorbent assay and amplified sensitive enzyme linked immunosorbent assay kits (Biosource Europe SA, Nivelles, Belgium), as described previously. ${ }^{11}$

\section{Skin specimens}

Skin biopsies $(5 \mathrm{~mm})$ were obtained from the forearm lesions (punch biopsies). The specimens were divided into two parts, one was formalin fixed and paraffin wax

Table 1 Clinical features of the patients with systemic sclerosis and the healthy controls

\begin{tabular}{|c|c|c|c|c|c|}
\hline Variable & Total SSc $(n=19)$ & ISSc $(n=10)$ & dSSc $(n=9)$ & $\mathrm{HC}(n=6)$ & $\mathrm{p}$ Value (SSc $v \mathrm{HC})$ \\
\hline Age (years)* & $34.8(2.6)$ & $37.6(15.7)$ & $33.6(8.5)$ & $33.03(7.2)$ & NS \\
\hline Sex (female: male ratio) & $18: 1$ & $9: 1$ & $9: 0$ & $5: 1$ & NS \\
\hline Duration (years)* & $04.8(3.5)$ & $05.2(3.9)$ & $4.5(3.8)$ & - & - \\
\hline Arthralgia/Arthritis & $8 / 19(42.1 \%)$ & $04 / 10(40 \%)$ & $04 / 9(44 \%)$ & Absent & $<0.05$ \\
\hline Raynaud's phenomenon & $19 / 19(100 \%)$ & $10 / 10(100 \%)$ & $09 / 9(100 \%)$ & Absent & $<0.05$ \\
\hline \multicolumn{6}{|l|}{ Pulmonary functions* } \\
\hline FVC & $60.9(15.4)$ & $63.01(16.3)$ & $58.0(13.3)$ & $94.4(7.7)$ & $<0.05$ \\
\hline FEV1 & $66.1(16.1)$ & $54.4(22.1)$ & $55.0(13.9)$ & $95.2(10.3)$ & $<0.05$ \\
\hline FEVI/FVC & 91.5 (10.4) & $92.1(6.6)$ & 86.5 (16.8) & $97.5(6.9)$ & NS \\
\hline Proteinuria & $3 / 19(15.8 \%)$ & $01 / 10(10 \%)$ & $02 / 9(22 \%)$ & Absent & $<0.05$ \\
\hline \multicolumn{6}{|l|}{$\mathrm{ESR}^{*}$} \\
\hline First hour & $27.4(16.3)$ & $30.5(19.5)$ & $25.4(15.2)$ & $08.5(5.1)$ & $<0.05$ \\
\hline Second hour & $52.0(22.1)$ & $54.7(3)$ & $50.3(20.2)$ & $13.3(20.1)$ & $<0.05$ \\
\hline Positive RF & $05 / 19(26.3 \%)$ & $03 / 10(30 \%)$ & $02 / 9(22 \%)$ & Absent & $<0.05$ \\
\hline Positive CRP & $05 / 19(26.3 \%)$ & $02 / 10(20 \%)$ & $03 / 9(33 \%)$ & Absent & $<0.05$ \\
\hline Positive ANA & $19 / 19(100 \%)$ & $10 / 10(100 \%)$ & $09 / 9(100 \%)$ & Absent & $<0.05$ \\
\hline
\end{tabular}

ANA, antinuclear antibody; CRP, C reactive protein; dSSc, diffuse systemic sclerosis; ESR, erythrocytic sedimentation rate; $F$, female; $F E V 1$, forced expiratory volume; FVC, forced vital capacity; HC, healthy controls; ISSc, limited systemic sclerosis; M, male; RF, rheumatoid factor; SSc, systemic sclerosis. *Values are mean (SD). 
Table 2 Alterations of the mononuclear inflammatory cells in the BALF, blood, and the skin of patients with SSc and healthy controls

\begin{tabular}{|c|c|c|c|c|c|}
\hline & $\operatorname{SSc}(n=19)$ & ISSc $(n=10)$ & dSSc $(n=9)$ & $H C(n=6)$ & p Value \\
\hline \multicolumn{6}{|l|}{ BALF } \\
\hline Total & $238(49.8)$ & $214(32.8)$ & $260(53.7)$ & 157.5159 .40 & $<0.05$ \\
\hline Monocytes & $80.7(3.2)$ & $81.2(4.5)$ & $79.6(8.8)$ & $90.2(1.5)$ & NS \\
\hline Lymphocytes & $13.5(1.3)$ & $14.2(4.1)$ & $12.9(5.8)$ & $6.5(2.1)$ & $<0.05$ \\
\hline Neutrophils & $4.3(0.6)$ & $4.1(1.2)$ & $4.7(2.0)$ & $2.2(0.3)$ & $<0.05$ \\
\hline Eosinophils & $1.5(1.6)$ & $1.1(0.6)$ & $1.1(0.9)$ & $1.1(0.6)$ & NS \\
\hline \multicolumn{6}{|l|}{ Blood } \\
\hline Total & $8.3(3.1)$ & $8.3(3.8)$ & $8.3(2.5)$ & $6.1(1.01)$ & NS \\
\hline Monocytes & $5.0(3.6)$ & 4.1 (2.2) & $5.9(4.6)$ & 4.7 (1.2) & NS \\
\hline Lymphocytes & $37.3(15.8)$ & $42.0(14.5)$ & $32.1(16.4)$ & $40.8(5.8)$ & NS \\
\hline Neutrophils & $52.0(18.5)$ & $46.4(16.8)$ & $57.6(19.6)$ & $46.5(7.7)$ & NS \\
\hline Eosinophils & $5.8(3.8)$ & $7.4(3.4)$ & $4.1(3.8)$ & $1.0(1.0)$ & $<0.05$ \\
\hline \multicolumn{6}{|l|}{ Skin } \\
\hline Total & $3.9(3.6)$ & $3.6(3.4)$ & $4.3(4.0)$ & $2.1(1.7)$ & NS \\
\hline Histiocytes & 10.1 (3.9) & $10.9(4.4)$ & $10.7(4.2)$ & $2.0(0.3)$ & $<0.05$ \\
\hline Lymphocytes & $4.2(1.8)$ & $2.9(2.2)$ & $5.8(1.7)$ & $7.5(1.8)$ & $<0.05$ \\
\hline Neutrophils & $0.3(0.3)$ & $0.3(0.3)$ & $0.3(0.3)$ & $0.0(0.0)$ & $<0.05$ \\
\hline Eosinophils & $0.3(0.2)$ & $0.3(0.3)$ & $0.3(0.3)$ & $0.0(0.0)$ & $<0.05$ \\
\hline
\end{tabular}

Values are mean (SD).

The $\mathrm{p}$ values are for patients with SSc compared with HCs.

BALF, bronchoalveolar lavage fluid; dSSc, diffuse systemic sclerosis; HC, healthy controls; ISSc, limited systemic sclerosis; SSc, systemic sclerosis.

embedded, whereas the other portion was processed for frozen sections.

\section{Evaluation of MICs in the skin}

The position and extent of the MIC infiltrates were analysed in the skin lesions. The cells were evaluated histologically in haematoxylin and eosin stained sections at the lesional stroma, perivascular areas, the overlying epidermis, and the subcutaneous tissues. Lymphocytic infiltration was determined by light microscopy with a $\times 100$ oil immersion lens in at least three different fields, and the mean values were calculated.

\section{Immunohistochemical analysis of CD4+ and CD8+ T cells in BALF, blood films, and skin}

Immunostaining was carried out as described previously. ${ }^{11}$ Briefly, sections mounted on glass slides were dewaxed and rehydrated through graded alcohols to water. Endogenous peroxidase activity was blocked with $0.6 \%$ $\mathrm{H}_{2} \mathrm{O}_{2}$ in methanol. Sections were then immersed in antigen retrieval solution (10mM sodium citrate buffer, $\mathrm{pH} 6.0)$ and subjected to enzymatic digestion $(0.1 \%$ trypsin $)$ for 20 minutes. Non-specific protein binding was blocked with a 10 minute exposure to $10 \%$ normal goat serum. Sections were then incubated with mouse monoclonal antibodies for 30 minutes at room temperature. After brief rinsing in PBS, the avidin-biotin-peroxidase complex method was used according to the manufacturer's instructions. Sections were next treated with peroxidase labelled streptavidin for 30 minutes at room temperature and incubated with 1,4-diaminobenzidine and $0.06 \% \mathrm{H}_{2} \mathrm{O}_{2}$ for five minutes. They were counterstained with haematoxylin, dehydrated, cleared, and mounted under coverslips. Frozen tissue sections were used for the anti-CD4 antibody, using the same procedure except for the antigen retrieval steps. ${ }^{12}$ The skin sections were stained with CD20 (pan-B cell marker). The results (CD4/CD8 counts) were expressed as a percentage.

\section{Positive controls}

Known $\mathrm{B}$ and $\mathrm{T}$ cell lymphomas and tonsils were used as positive controls for CD20, CD3, CD4, and CD8 immunostaining.

\section{Negative controls}

Additional sections of lesional skin and the positive controls were stained in parallel, but with omission of the primary antibody. ${ }^{12}$

\section{Statistical analysis}

Statistical analysis was carried out using Spearman's rank test and the Mann-Whitney U test. The results are presented as mean (SD). p Values $<0.05$ were considered significant.

\section{RESULTS}

\section{Clinical characteristics of the patients with SSc}

The patients with SSc were slightly older (mean age, 34.8 years; SD, 11.47) than the HCs (mean, 33.6 years; SD, 2.6). There was a significant predilection for female sex in the patient group (F: M ratio, $18: 1 ; p<0.0001$ ). The mean (SD) duration of the disease process was 4.76 (3.49) years. Arthralgia, Raynaud's phenomenon, ECG changes (right ventricular hypertrophy, bundle branch block, and ventricular extrasystole), and renal changes were seen in 18, 19, seven, and three of the patients, respectively. Positive rheumatoid factor and antinuclear antibodies were seen in five and 19 of the patients with SSc, respectively. The CRP concentration was more than $6 \mathrm{mg} /$ litre in five patients. Deterioration in pulmonary function was only pronounced late in the disease. High resolution computerised tomography (HRCT) showed more deterioration in patients with dSSc than in those with 1SSc. In dSSc, HRCT revealed ground glass, honeycombing, and mixed patterns in five, one, and three patients, respectively. In contrast, in ISSc HRCT revealed normal, ground glass, and honeycombing patterns in six, three, and one patients, respectively. Moreover, $x$ rays revealed a normal pattern in nine patients with ISSc and one with dSSc. A reticular pattern was seen in one patient with ISSc and eight patients with dSSc. None of these abnormalities was seen in the HCs. Deterioration of pulmonary and renal function was more common in patients with dSSc than in those with ISSc. Right ventricular systolic dimensions were within the normal range for all participants. Table 1 shows a summary of these results.

\section{Alterations of MICs in BALF, peripheral blood, and skin lesions in SSc}

Compared with HCs, BALF from patients with SSc had significantly higher total and some differential MIC counts 
Table 3 Alterations of T cells and cytokines in the patients with SSc and HCs

\begin{tabular}{|c|c|c|c|c|c|}
\hline & Total SSc $(n=19)$ & ISSc $(n=10)$ & dSSc (n=9) & HCs $(n=6)$ & p Value \\
\hline CD4+ cells in blood & $63(3.3)^{*}$ & $59.9(3.0)$ & $65.1(3.9)^{*}$ & $54.3(3.1)$ & $<0.05$ \\
\hline CD8+ cells in blood & $22.7(3.4)$ & $26.8(3.6)$ & $28.8(3.2)$ & $28.9(9.3)$ & NS \\
\hline CD4/CD8 ratio in blood & $2.8(0.2)$ & $2.2(0.2)$ & $2.3(0.3)$ & $1.8(0.8)$ & $<0.05$ \\
\hline TNF $\alpha$ concentration in serum $(\mathrm{pg} / \mathrm{ml})$ & $38.6(7.6)^{\star *}$ & $40.1(6.8)^{\star \star}$ & $36.9(8.4)^{\star *}$ & $7.5(2.1)$ & $<0.01$ \\
\hline $\mathrm{IL}-1 \beta$ concentration in serum $(\mathrm{pg} / \mathrm{ml})$ & $21.6(9.4)^{\star *}$ & $23.4(10.9)^{\star *}$ & $19.7(7.6)^{\star \star}$ & $9.2(6.2)$ & $<0.05$ \\
\hline CD4+ cells in BALF & $51.1(4.8)$ & $50.7(4.8)$ & $51.6(5.3)$ & $51.9(4.4)$ & NS \\
\hline CD8+ cells in BALF & $35.7(2.6)^{*}$ & $36.2(2.6)^{*}$ & $35.2(2.6)^{*}$ & $25.1(3.1)$ & $<0.05$ \\
\hline CD4/CD8 ratio in BALF & $1.4(0.1)^{\star *}$ & $1.3(0.1)^{* *}$ & $1.4(0.1)^{\star *}$ & $2.1(0.2)$ & $<0.01$ \\
\hline TNF $\alpha$ concentration in BALF (pg/ml) & $136.7(50.7)^{\star * *}$ & $147.1(53.4)^{\star * \star}$ & $125.1(47.5)^{\star * \star} \dagger$ & $41.1(34.4)$ & $<0.001$ \\
\hline IL-1 $\beta$ concentration in BALF (pg/ml) & $49.4(11.9)^{* *}$ & $51.2(11.6)^{* *}$ & $47.5(12.3)^{* *}$ & $11.3(11.5)$ & $<0.01$ \\
\hline
\end{tabular}

Values are mean (SD).

The $p$ values are for patients with SSc compared with HCs.

For comparison with the control group: ${ }^{*} p<0.05,{ }^{* *} p<0.01,{ }^{* * *} p<0.001$. For comparison between diffuse and limited SSc: $t p<0.05$.

BALF, bronchoalveolar lavage fluid; dSSc, diffuse systemic sclerosis; HC, healthy controls; IL-1 $\beta$, interleukin 1 $\beta$; ISSc, limited systemic sclerosis; SSc, systemic sclerosis; TNF $\alpha$, tumour necrosis factor $\alpha$.

$(238(49.8) v 157.5(59.4), 80.7$ (3.3) v $90.2(1.5), 13.5(1.3) v$ $6.5(2.1), 4.3(0.6) \vee 2.2(0.3)$, and $1.5(1.6) \vee 1.1(0.6)$ for total MICs, alveolar macrophages, lymphocytes, neutrophils, and eosinophils, respectively; $\mathrm{p}<0.05)$. In addition, CD8+ T cell counts were significantly higher in the patients with SSc than in HCs (35.7 (2.6) v 25.1 (3.1); p < 0.05). In contrast, the CD4/CD8 ratio in BALF was significantly lower in patients with SSc than in HCs $(1.4(0.1) v 2.1(0.2) ; \mathrm{p}<0.01)$. In the peripheral blood, MIC values were higher in the patients with SSc than in HCs $(8.3$ (3.1) v 6.1 (1.01), 5.0 (3.6) v 4.7 (1.2), 37.3 (15.8) v 40.8 (5.8), 52.0 (18.5) v 46.5 (7.7), and 5.8 (3.8) $v 1.0$ (1.0) for total MICs, blood monocytes, lymphocytes, neutrophils, and eosinophils, respectively). In the skin, there were significantly increased numbers of histiocytes, eosinophils, and neutrophils in the lesional skin of patients with SSc compared with the normal skin of the HCs (10.1 (3.9) $v$ $2.0(0.3), 0.3(0.2) \vee 0.0$, and $0.3(0.3) \vee 0.0$, respectively; $\mathrm{p}<0.05)$. In contrast, significantly fewer lymphocytes were seen in the lesional skin of patients with SSc than in the HCs (4.2 (1.8) v 7.5 (1.8), respectively; $\mathrm{p}<0.05)$. The lymphocytes and macrophages were seen between collagen bundles around the skin adenexa and the blood vessels (perivascular).

Patients with dSSc had higher total MIC counts and also some higher differential MIC counts in BALF, blood, and skin than those with ISSc. In BALF, there were higher total and differential MICs counts in dSSc than in ISSc (260 (53.7) $v$ 214 (32.8), $79.6(8.8) v 81.2(4.5), 12.9$ (5.8) v 14.2 (4.1), 4.7 (2.0) $v 4.1(1.2), 1.1(0.9) \vee 1.1(0.6)$ for total MIC, alveolar macrophages, lymphocytes, neutrophils, and eosinophils, respectively), although the results were not significant. In the peripheral blood, some MIC values were higher in patients with dSSc than in those with ISSc $(8.3(2.5) v 8.3$ (3.8), 5.9 (4.6) v 4.1 (2.2), 32.1 (16.4) $v 42.0$ (14.5), 57.6

Table 4 Comparison between T cell subsets and cytokine production in BALF and blood in patients with systemic sclerosis

\begin{tabular}{llll}
\hline & Blood & BALF & p Value \\
\hline CD4+ cell count & $63.0(3.3)$ & $51.1(4.9)$ & $<0.05$ \\
CD8+ cell count & $22.7(3.4)$ & $35.7(2.6)$ & $<0.01$ \\
CD4/CD8 ratio & $2.8(0.2)$ & $1.4(0.1)$ & $<0.01$ \\
TNF $\alpha$ (pg/ml) & $38.6(7.6)$ & $136.8(50.7)$ & $<0.001$ \\
IL-1 $\beta$ (pg/ml) & $21.6(9.4)$ & $49.5(11.9)$ & $<0.01$ \\
\hline
\end{tabular}

Values are mean (SD).

CD8+ T cell counts, TNF $\alpha$, and IL-1 $\beta$ were significantly higher in patients' BALF than in blood $(p<0.01, p<0.001$, and $p<0.01$, respectively). Moreover, $C D 4+T$ cell counts the $C D 4 / C D 8$ ratio in BALF were significantly lower than in blood $(p<0.05$ and $p<0.01$, respectively). BALF, bronchoalveolar lavage fluid; IL-1 $\beta$, interleukin $1 \beta ; \mathrm{TNF} \alpha$, tumour necrosis factor $\alpha$.
(19.6) v 46.4 (16.8), and 4.14 (3.8) v 7.4 (3.4) for total MIC, blood monocytes, lymphocytes, neutrophils, and eosinophils, respectively. However, these differences were not significant except for the eosinophils (7.4 (3.4) $v 4.1$ (3.8) for ISSc and dSSc, respectively; $\mathrm{p}<0.05$ ).

In the skin, there were more lymphocytes in patients with dSSc than in those with ISSc $(4.3(4.0) v 3.6(3.4), 10.7(4.2) v$ 10.9 (4.4), $5.8(1.7) \vee 2.9(2.2), 0.3(0.3) \vee 0.3(0.3)$ and 0.3 (0.3) $v 0.3(0.3)$ for total MIC, histiocytes, lymphocytes, neutrophils, and eosinophils, respectively). Table 2 shows a summary of these results.

\section{Increased cytokine concentrations in the BALF and peripheral blood of patients with SSc}

Patients with SSc had significantly higher TNF $\alpha$ and IL-1 $\beta$ concentrations $(\mathrm{pg} / \mathrm{ml})$ than HCs in BALF (136.7 (50.7) $v 41.1$ (34.4) and $49.4(11.9) v 11.3$ (11.5), respectively; $p<0.001$ and $\mathrm{p}<0.05)$ and peripheral blood (38.6 (7.6) $\vee 7.5(2.1)$ and $21.6(9.4) \vee 9.2(6.2)$, respectively; $\mathrm{p}<0.01$ for both).

Patients with dSSc had lower TNF $\alpha$ and IL-1 $\beta$ concentrations in BALF and peripheral blood than those with ISSc, although the results were not significant except for the TNF $\alpha$ values in BALF $(\mathrm{p}<0.05)$ (BALF: 125.1 (47.5) v 147.1 (53.4), 47.5 (12.3) v 51.2 (11.6), respectively; peripheral blood: (36.9 (8.4) $v 40.1(6.8), 19.7$ (7.6) $\vee 23.4$ (10.9), respectively). Table 3 shows a summary of these results.

\section{Alterations of CD4/CD8+ T cells, IL- $1 \beta$, and TNF $\alpha$ in BALF and the peripheral blood in patients with SSC} Compared with HCs, patients with SSc had significantly higher: (1) TNF $\alpha$ concentrations (136.7 (50.7) v 41.1 (34.4); $\mathrm{p}<0.001$ ), (2) IL-1 $\beta$ concentrations (49.4 (11.9) $v 11.3$ (11.5); p < 0.01), and (3) CD8+ T cell counts (35.7 (2.6) v 25.1 (3.1), $\mathrm{p}<0.05)$ in BALF. In contrast, CD4+ T cell counts and CD4/CD8 ratios were lower in the BALF of patients with SSc than in HCs (51.1 (4.8) $v 51.9$ (4.4) and $1.4(0.1) \vee 2.1$ (0.2), respectively). In the peripheral blood, the CD4 counts and CD4/CD8 ratios were higher in patients with SSc than in

Table 5 Correlation between FVC, T cell subsets, and the cytokine profile in patients with systemic sclerosis

\begin{tabular}{lll}
\hline Feature & $r$ Value & $p$ Value \\
\hline CD8+ cells in BALF & 0.4 & $<0.05$ \\
TNF $\alpha$ in BALF $(p g / m l)$ & -0.7 & $<0.001$ \\
IL- $1 \beta$ in BALF $(\mathrm{pg} / \mathrm{ml})$ & -0.6 & $<0.01$ \\
\hline
\end{tabular}

BALF, bronchoalveolar lavage fluid; FVC, forced vital capacity; IL-1 $\beta$, interleukin $1 \beta$; TNF $\alpha$, tumour necrosis factor $\alpha$. 
HCs $(63(3.3) v 54.3$ (3.1) and $2.8(0.2) v 1.8(0.8) ; \mathrm{p}<0.05$ and $\mathrm{p}<0.01$, respectively).

Compared with dSSc, patients with ISSc had higher: (1) TNF $\alpha$ in BALF (147.1 (53.4) v 125 (47.5)) and in peripheral blood (40.1 (6.8) v 36.9 (8.4)); (2) IL-1 $\beta$ in BALF (51.2 (11.6) $v 47.5$ (12.3)) and peripheral blood (23.4 (10.9) v 19.7 (7.6); and (3) CD8+ cells in BALF (36.2 (2.6) $v 35.2$ (2.6)). In contrast, compared with ISSc, patients with dSSc had higher: (1) CD4 counts (65.1 (3.9) v 59.9 (3.0)), CD8 counts (28.8 $(3.2) v 26.8(3.6))$, and CD4/CD8 ratios $(2.3(0.3) \vee 2.2(0.2))$ in the peripheral blood; (2) CD4 counts (51.6 (5.3) $\vee 50.7$ $(4.8))$ and CD4/CD8 ratios ( $1.4(0.1) v 1.3(0.1))$ in BALF; and (3) lower CD8+ T cell counts in BALF (35.2 (2.6) v $36.2(2.6))$. However, these differences were significant only for TNF $\alpha$ in BALF $(p<0.05)$. Table 3 shows a summary of these results.

\section{Correlations between CD8 + T cells, TNF $\alpha$, and IL- $1 \beta$ in BALF and FVC}

There was a significant inverse correlation between $\mathrm{TNF} \alpha$, and IL-1 $\beta$ and FVC $(r=-0.7, \mathrm{p}<0.001 ; r-0.5, \mathrm{p}<0.01$, respectively). Table 5 summarises these results.

\section{Histological changes in the skin}

The skin biopsies were examined by two certified pathologists (Drs H Ismael and M R Hussein). The histological changes included variable atrophy of the epidermis, loss of the rete ridges, skin appendages, and pronounced deposition of collagen bundles in the papillary and reticular dermis (fig 1). A MIC infiltrate was seen around the blood vessels. It was formed predominantly of lymphocytes, histiocytes, occasional eosinophils, and neutrophils. Further immunohistochemical analysis revealed that the lymphocytes lacked CD20 reactivity.

\section{DISCUSSION}

Systemic sclerosis is an autoimmune disease characterised by microvascular involvement and fibrosis affecting the skin, lung, and heart. To date, our understanding of the changes in MICs, T cells, and cytokines in SSc is still incomplete. In our study, we hypothesised that SSc is associated with alterations in MICs, $\mathrm{T}$ cell subsets (CD4/CD8), and cytokine production in the lung, blood, and skin. We also hypothesised that these alterations contribute to the genesis of the pulmonary and skin lesions and would therefore correlate with the clinicopathological characteristics of these lesions. We carried out this investigation to fill this existing gap in the literature and to test our hypotheses. Therefore, we examined 19 patients with SSc and six matched healthy individuals. Our results clearly showed the following: (1) SSc has a predilection for middle aged women; (2) MICs and cytokine concentrations (IL-1 $\beta$ and TNF $\alpha$ ) are increased in the BALF, blood, and skin lesions of patients with SSc, (3) altered CD4 and CD8 T cell counts are seen in the BALF, blood, and skin of patients with SSc; and (5) there is a significant correlation between CD8+ T cell counts and cytokine concentrations in BALF and pulmonary function (FVC).

In agreement with previous studies, SSc in our series was associated with: (1) a predilection for the female sex, with an average age of 34.59 years; (2) pronounced restriction of cardiopulmonary and renal functions late in the disease; and (3) the presence of positive rheumatoid factor and antinuclear antibodies. ${ }^{13}$ The female sex predilection may result from an underlying genetic predisposition, probably characterised by a single inherited dominant allele on the $\mathrm{X}$ chromosome. ${ }^{14}$ The raised CRP concentration is probably the result of the increased production of IL- $1 \beta$ and $\mathrm{TNF} \alpha$, which can stimulate the production of this protein by hepatocytes. In addition, the finding of raised CRP may reflect underlying endothelial dysfunction or possible development of rheumatoid arthritis in these patients. ${ }^{15}$ The finding of positive rheumatoid factor and antinuclear antibodies may reflect an ongoing autoimmune response. However, whether this response represents a primary abnormality or is secondary to tissue damage is still unknown. ${ }^{13} 14$

In agreement with other groups, ${ }^{76-28}$ we found higher MIC counts in the BALF, blood, and skin lesions of patients with SSc than in HCs. Furthermore, higher neutrophil, eosinophil, lymphocyte, and histiocyte cell counts were seen in the patients with SSc. Thus, we propose that these MICs operate by several mechanisms, either individually or together, in the development of the skin and lung lesions of SSc. First, the presence of neutrophils and eosinophils in BALF contributes to the ongoing alveolitis in these patients. In this respect, the increased numbers of neutrophils in the skin and BALF might be the result of their increased random motility, which in turn might be caused by inhibition of inactivators of chemotactic factors and circulating cell directed inhibitors of chemotaxis in the sera of patients with SSc. ${ }^{29}$ Second, the presence of chronic inflammatory cells (lymphocytes, eosinophils, and histiocytes) in the local milieu of the blood, BALF, and skin suggests some role for chronic inflammation in the derangement of pulmonary and cutaneous structures in SSc. Third, the histiocytes, eosinophils, and lymphocytes in the BALF and skin contribute to the pulmonary and skin fibrosis in these patients. In this regard, the macrophages, lymphocytes, and eosinophils represent potential sources of profibrotic cytokines (IL- $1 \beta$, IL-6, TNF $\alpha$, and transforming growth factor $\beta$ (TGF $\beta)$ ) and fibronectin. ${ }^{14}{ }^{30}$ These molecules can induce pulmonary and skin fibrosis by several mechanisms. First, fibronectin can contribute to fibrosis by attracting fibroblasts, stabilising collagen fibres, and preventing collagen degradation by collagenase enzymes. ${ }^{31}$ In addition, fibronectin binds to monocytes and stimulates them to

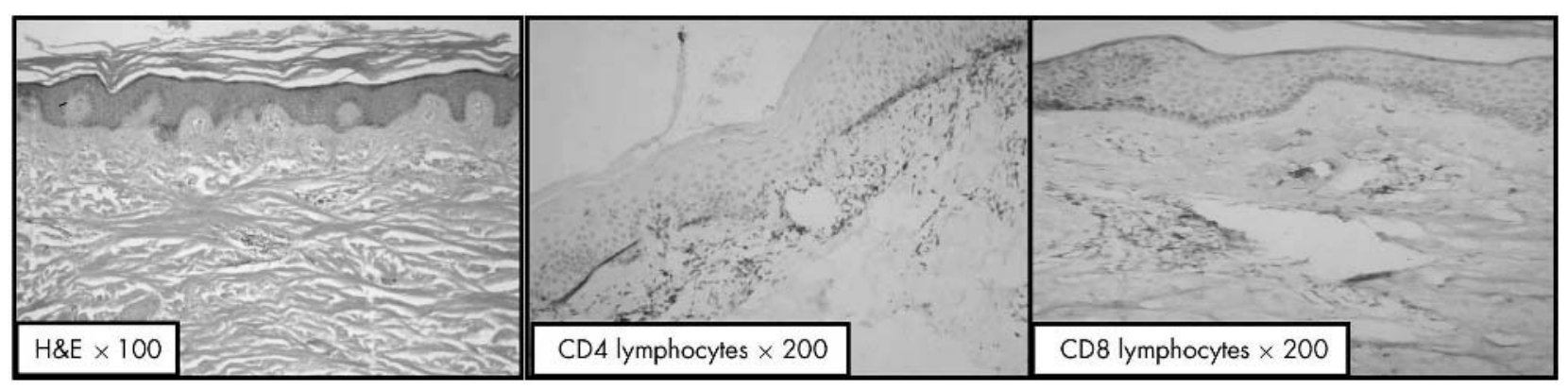

Figure 1 Haematoxylin and eosin (H\&E) grade II scleroderma showing encroachment of the collagen on the adenexal structures and loss of definition between the papillary and reticular dermis (original magnification, $\times 100$ ), abundance of CD4+ T cells in the dermis (original magnification, $\times 200$ ), and scarcity of CD8+ T cells in the dermis (original magnification, $\times 200$ ). 
produce more growth factors. ${ }^{32}{ }^{33}$ Second, TGF $\beta$ is a potent fibroblast mitogen and may potentiate the effects of other fibroblast growth factors. It stimulates fibroblasts to increase their synthesis of extracellular matrix proteins and inhibits the synthesis of collagenase. The net effect is to promote matrix formation and fibrosis. In support of our proposition, administration of anti-TGF $\beta$ antibodies can inhibit bleomycin induces lung fibrosis in the SSc animal model. ${ }^{34}$ Moreover, fibronectin in SSc may represent an abnormal fibroblast response to the increased number of MICs.

"The ability to detect alterations in mononuclear inflammatory cells, T cells, and cytokines in bronchoalveolar lavage fluid highlight its value in assessing ongoing lung disease (alveolitis), monitoring its course, and choosing the appropriate treatment"

The predominance of histiocytes in the dermal infiltrate not only agrees with the previous investigations, ${ }^{22}{ }^{35}$ but also suggests their possible role in the pathogenesis of these lesions. Because these cells are capable of producing various cytokines, including $\mathrm{TNF} \alpha{ }^{36}$ we propose that their accumulation plays a role in modulating the function of human dermal fibroblasts in a way that enhances fibrogenesis. Of note, high eosinophil cell counts were found in the skin, BALF, and blood of patients with SSc compared with HCs. We propose that these increased eosinophil counts contribute to the pulmonary and skin fibrosis by several mechanisms. First, eosinophils are potential sources of TGF $\beta{ }^{37}$ Second, eosinophils have direct modulatory effects on fibroblast proliferation and collagen synthesis. ${ }^{38}$ Third, eosinophils are major sources of several key profibrogenic cytokines that stimulate fibroblasts. In support of this proposition, previous studies have reported a correlation between lung eosinophil counts and poor prognosis and the lack of responsiveness to treatment, ${ }^{39}{ }^{40}$ and the fact that eosinophils contain eosinophil cationic protein, which promotes inflammation and modulates fibroblast-collagen interactions.$^{41}$ Moreover, recent reports indicated that lymphocytes can stimulate fibroblast proliferation and therefore increase collagen synthesis. ${ }^{42}$ These experimental data also suggest a role for lymphocytes in the genesis of fibrosis in the lung and skin. ${ }^{42}$

In agreement with previous reports, ${ }^{36}{ }^{16-45}$ there were significantly higher cytokine concentrations in the BALF and peripheral blood of patients with SSc than in HCs. These increased concentrations probably result from the recruitment of macrophages and $\mathrm{T}$ cells to the lung and skin and their subsequent activation. These cytokines modulate the fibrotic process in these organs. ${ }^{42}$ In this regard, IL- $1 \beta$ and $\mathrm{TNF} \alpha$ have inflammatory effects on the endothelium, which is the initial target in the SSc disease process. Damage to the endothelium may also initiate the fibrotic process, either through the effects of ischaemia or through growth modulating mediators released from inflammatory cells. ${ }^{46}$ In addition, IL- $1 \beta$ can induce the synthesis of glycosaminoglycan in fibroblasts and therefore enhance fibrosis. ${ }^{47} 48$

The increased CD4+ T cell counts and CD4/CD8 ratio in the peripheral blood and skin of patients with SSc agree with previous studies. ${ }^{22} 3549$ This increased CD4/CD8 ratio may be the result of recruitment of increased numbers of CD4+ cells coupled with decreased CD8+ T cell counts, and/or the effects of disease duration. Of note, the skin infiltrate was pronounced during the inductive stage of the disease and gradually disappeared with transition to the sclerotic stage. Thus, it is possible that the increased numbers of CD4+ cells may release profibrotic cytokines such as IL-1 $\beta$, IL-6, TNF $\alpha$, and TGF $\beta$, which play a pivotal role in the genesis of fibrosis in patients with SSc. Alternatively, the increased CD8+ cell
Take home messages

- Patients with systemic sclerosis show alterations in mononuclear inflammatory cells, $T$ cell subsets, and cytokine (interleukin $1 \beta$ and tumour necrosis factor $\alpha$ ) production in their bronchoalveolar lavage fluid, blood, and skin

- These alterations correlate with the ongoing lung and skin disease and the clinicopathological characteristics of the lesions, and might be useful for monitoring disease activity and as a guide to further therapeutic intervention

- Further investigations are recommended to reveal the role of other molecules, such as transforming growth factor $\beta$ and fibronectin, in the development of fibrosis in these lesions

counts and decreased CD4/CD8 ratio seen in the BALF of patients with SSc also concur with previous studies, ${ }^{17-20} 4445$ and are probably the result of decreased in CD4+ T cell counts and increased numbers of CD8+ cells.

There were inverse correlations between CD8+ T cell counts and cytokine concentrations in BALF and pulmonary function (FVC). These findings suggest: (1) the involvement of $\mathrm{T}$ cell mediated immunity in the perpetuation of SSc, ${ }^{50}{ }^{51}(2)$ a common mechanism regulating the production of these cytokines in patients with SSc, and (3) that the evaluation of IL- $1 \beta, \mathrm{TNF} \alpha$, and CD8 T cell counts would be useful in the clinical evaluation of disease activity and future outcome. Of note, several studies revealed consistent findings for BALF and lung biopsy. ${ }^{33}$ Thus, the ability to detect alterations in MICs, $\mathrm{T}$ cells, and cytokines in BALF highlight its value in assessing ongoing lung disease (alveolitis), monitoring its course, and choosing the appropriate treatment.

To summarise, SSc is associated with alterations in MICs, $\mathrm{T}$ cells, and cytokine production in the BALF, blood, and skin. These alterations correlate with the ongoing lung and skin disease and the clinicopathological characteristics of the lesions. Therefore, these alterations may prove useful in monitoring disease activity and as a guide to further therapeutic intervention. Further investigations are recommended to reveal the role of other molecules, such as TGF $\beta$ and fibronectin, in the genesis of fibrosis in these lesions.

\section{Authors' affiliations}

M R Hussein, H I Hassan, Department of Pathology, Faculty of Medicine, Assiut University, Assiut, Egypt

M Elkholy, Department of Respiratory Medicine, Faculty of Medicine, Assiut University

E R M Hofny, Department of Dermatology and Venereology, Assiut University

N A Fatehy, Department of Rheumatology and Rehabilitation, Assiut University

A E A Abd Elmoniem, Department of Internal Medicine, Faculty of Medicine, Assiut University

A M Ezz El-Din, O A Afifi, H G Rashed, Department of Clinical Pathology, Assiut University

\section{REFERENCES}

1 Mayes MD, Lacey JV Jr, Beebe-Dimmer J, et al. Prevalence, incidence, survival, and disease characteristics of systemic sclerosis in a large US population. Arthritis Rheum 2003:48:2246-55.

2 Gruschwitz MS, Albrecht M, Vieth G, et al. In situ expression and serum levels of tumor necrosis factor-alpha receptors in patients with early stages of systemic sclerosis. J Rheumatol 1997;24:1936-43. 
3 Fujii $\mathbf{H}$, Hasegawa M, Takehara $\mathrm{K}$, et al. Abnormal expression of intracellular cytokines and chemokine receptors in peripheral blood T lymphocytes from patients with systemic sclerosis. Clin Exp Immunol 2002; 130:548-56.

4 Lonzetti LS, Joyal F, Raynauld JP, et al. Updating the American College of Rheumatology preliminary classification criteria for systemic sclerosis: addition of severe nailfold capillaroscopy abnormalities markedly increases the sensitivity for limited scleroderma. Arthritis Rheum $2001 ; 44: 735-6$.

5 Pantelidis P, Fanning GC, Wells AU, et al. Analysis of tumor necrosis factoralpha, lymphotoxin-alpha, tumor necrosis factor receptor II, and interleukin-6 polymorphisms in patients with idiopathic pulmonary fibrosis. Am J Respir Crit Care Med 2001;163:1432-6.

6 Boyum A. Isolation of lymphocytes, granulocytes and macrophages. Scand J Immunol 1976;5(suppl):9-15.

7 Gudbjornsson B, Hallgren R, Nettelbladt O, et al. Phenotypic and functional activation of alveolar macrophages, $T$ lymphocytes and NK cells in patients with systemic sclerosis and primary Sjogren's syndrome. Ann Rheum Dis 1994;53:574-9

8 Farr AG, Nakane PK. Immunohistochemistry with enzyme labeled antibodies: a brief review. J Immunol Methods 1981;47:129-44.

9 Frigieri L, Mormile F, Grilli N, et al. Bilateral bronchoalveolar lavage in progressive systemic sclerosis: interlobar variability, lymphocyte subpopulations, and functional correlations. Respiration 1991;58:132-40.

10 Mason DY, Cordell JL, Gaulard P, et al. Immunohistological detection of human cytotoxic/suppressor T cells using antibodies to a CD8 peptide sequence. J Clin Pathol 1992;45:1084-8.

11 Hamilton S, Healy M, Corris P, et al. An immunohistochemical method for the detection of tumour necrosis factor alpha in cytospins of human bronchoalveolar lavage cells. Histochem J 1995;27:487-93.

12 Hussein MR, Sun M, Roggero E, et al. Loss of heterozygosity, microsatellite instability, and mismatch repair protein alterations in the radial growth phase of cutaneous malignant melanomas. Mol Carcinog 2002;34:35-44.

13 Al-Adhadh RN, Al-Sayed TA. Clinical features of systemic sclerosis. Saudi Med J 2001;22:333-6.

14 Rowell NR. Systemic sclerosis. The Watson Smith lecture 1984. J R Coll Physicians Lond 1985;19:23-30.

15 Yap SH, Moshage HJ, Hazenberg BP, et al. Tumor necrosis factor (TNF) inhibits interleukin (IL)-1 and/or IL-6 stimulated synthesis of C-reactive protein (CRP) and serum amyloid A (SAA) in primary cultures of human hepatocytes. Biochim Biophys Acta 1991;1091:405-8.

16 Salaffi $F$, Subiaco S, Carotti $M$, et al. Pulmonary inflammation in systemic sclerosis. An assessment by bronchoalveolar lavage. Recent Prog Med 1994:85:475-80.

17 Domagala-Kulawik J, Hoser G, Kawalec M, et al. Lymphocyte phenotyping in systemic sclerosis: a flow cytometry analysis of lymphocytes in bronchoalveolar lavage fluid. Anal Quant Cytol Histol 1997;19:264-70.

18 Domagala-Kulawik J, Hoser G, Doboszynska A, et al. Interstitial lung disease in systemic sclerosis: comparison of BALF lymphocyte phenotype and DLCO impairment. Respir Med 1998;92:1295-301.

19 Kopinski P, Korkosz M, Janowska E. Diagnosis of interstitial lung disease (ILD) in patients with systemic sclerosis. The significance of broncho-alveolar lavage (BAL)-personal observations. Folia Med Cracov 1996;37:51-66.

20 Kopinski P, Nalepa P, Wojas-Pelc A, et al. Changes in lymphocyte subsets in bronchoalveolar lavage fluid in patients with systemic sclerosis. Pol Merkuriusz Lek 2000;7:18-22.

21 Ercole LP, Malvezzi M, Boaretti AC, et al. Analysis of lymphocyte subpopulations in systemic sclerosis. I Investig Allergol Clin Immunol 2003;13:87-93.

22 Ishikawa O, Ishikawa H. Macrophage infiltration in the skin of patients with systemic sclerosis. J Rheumatol 1992;19:1202-6.

23 loanas $M$, lonita D, Cervis $L$, et al. Bronchoalveolar lavage findings in the pulmonary impairment related to systemic sclerosis. Pneumologia 2002:51:203-5.

24 Wells $A U$, Hansell DM, Rubens MB, et al. Fibrosing alveolitis in systemic sclerosis. Bronchoalveolar lavage findings in relation to computed tomographic appearance. Am J Respir Crit Care Med 1994;150:462-8.

25 Wells AU, Lorimer S, Majumdar S, et al. Fibrosing alveolitis in systemic sclerosis: increase in memory T-cells in lung interstitium. Eur Respir J 1995;8:266-71.

26 Wells AU, Hansell DM, Haslam PL, et al. Bronchoalveolar lavage cellularity: lone cryptogenic fibrosing alveolitis compared with the fibrosing alveolitis of systemic sclerosis. Am J Respir Crit Care Med 1998;157:1474-82.
27 Silver RM. Lymphokine activated killer (LAK) cell activity in the peripheral blood lymphocytes of systemic sclerosis (SSc) patients. Clin Exp Rheumatol 1990;8:481-6.

28 Harrison NK, McAnulty RJ, Haslam PL, et al. Evidence for protein oedema, neutrophil influx, and enhanced collagen production in lungs of patients with systemic sclerosis. Thorax 1990;45:606-10.

29 Minta JO, Kovacs EK, Sukenik S, et al. Chemotactic responsiveness and random motility of polymorphonuclear leukocytes from patients with progressive systemic sclerosis. J Rheumatol 1985;12:1125-8.

30 Cotton SA, Herrick AL, Jayson Ml, et al. TGF beta-a role in systemic sclerosis? J Pathol 1998;184:4-6.

31 Xu WD, Leroy EC, Smith EA. Fibronectin release by systemic sclerosis and normal dermal fibroblasts in response to TGF-beta. J Rheumatol 1991;18:241-6.

32 Silver RM, Miller KS. Lung involvement in systemic sclerosis. Rheum Dis Clin North Am 1990;16:199-216.

33 Rossi GA, Bitterman PB, Rennard SI, et al. Evidence for chronic inflammation as a component of the interstitial lung disease associated with progressive systemic sclerosis. Am Rev Respir Dis 1985;131:612-17.

34 Gruschwitz M, Muller PU, Sepp N, et al. Transcription and expression of transforming growth factor type beta in the skin of progressive systemic sclerosis: a mediator of fibrosis? J Invest Dermatol 1990:94:197-203.

35 Kraling BM, Maul GG, Jimenez SA. Mononuclear cellular infiltrates in clinically involved skin from patients with systemic sclerosis of recent onset predominantly consist of monocytes/macrophages. Pathobiology 1995;63:48-56.

36 Ferrarini M, Steen V, Medsger TA Jr, et al. Functional and phenotypic analysis of T lymphocytes cloned from the skin of patients with systemic sclerosis. Clin Exp Immunol 1990;79:346-52.

37 Ong VH, Evans LA, Shiwen X, et al. Monocyte chemoattractant protein 3 as a mediator of fibrosis: overexpression in systemic sclerosis and the type 1 tightskin mouse. Arthritis Rheum 2003;48:1979-91.

38 Levi-Schaffer F, Garbuzenko E, Rubin A, et al. Human eosinophils regulate human lung- and skin-derived fibroblast properties in vitro: a role for transforming growth factor beta (TGF-beta). Proc Natl Acad Sci U S A 1999;96:9660-5

39 Gharaee-Kermani $M$, Phan SH. The role of eosinophils in pulmonary fibrosis [review]. Int J Mol Med 1998;1:43-53.

40 Gharaee-Kermani M, McGarry B, Lukacs N, et al. The role of IL-5 in bleomycin-induced pulmonary fibrosis. J Leukoc Biol 1998;64:657-66.

41 Zagai U, Skold CM, Trulson A, et al. The effect of eosinophils on collagen gel contraction and implications for tissue remodelling. Clin Exp Immunol 2004; 135:427-33.

42 Huaux F, Liu T, McGarry B, et al. Dual roles of IL-4 in lung injury and fibrosis. $J$ Immunol 2003; 170:2083-92.

43 Umehara H, Kumagai S, Ishida $\mathrm{H}$, et al. Enhanced production of interleukin-2 in patients with progressive systemic sclerosis. Hyperactivity of CD4-positive T cells? Arthritis Rheum 1988;31:401-7.

44 Degiannis D, Seibold JR, Czarnecki M, et al. Soluble and cellular markers of immune activation in patients with systemic sclerosis. Clin Immunol Immunopathol 1990;56:259-70.

45 Degiannis D, Seibold JR, Czarnecki M, et al. Soluble interleukin-2 receptors in patients with systemic sclerosis. Clinical and laboratory correlations. Arthritis Rheum 1990;33:375-80.

46 Freemont AJ, Hoyland J, Fielding P, et al. Studies of the microvascular endothelium in uninvolved skin of patients with systemic sclerosis: direct evidence for a generalized microangiopathy. $\mathrm{Br} J$ Dermatol 1992; 126:561-8.

47 White B. Immunopathogenesis of systemic sclerosis. Rheum Dis Clin North Am 1996;22:695-708.

48 Akimoto $\mathrm{S}$, Hayashi $\mathrm{H}$, Ishikawa $\mathrm{H}$. Disaccharide analysis of the skin glycosaminoglycans in systemic sclerosis. Br J Dermatol 1992;126:29-34.

49 Tsuji-Yamada J, Nakazawa M, Minami M, et al. Increased frequency of interleukin 4 producing CD4+ and CD8+ cells in peripheral blood from patients with systemic sclerosis. J Rheumatol 2001;28:1252-8.

50 Gustafsson $\mathbf{R}$, Totterman TH, Klareskog L, et al. Increase in activated T cells and reduction in suppressor inducer T cells in systemic sclerosis. Ann Rheum Dis 1990;49:40-5.

51 Gustafsson R, Fredens K, Nettelbladt O, et al. Eosinophil activation in systemic sclerosis. Arthritis Rheum 1991;34:414-22. 